Z. Klin. Chem. Klin. Biochem.

13. Jg. 1975, S. $129-131$

\title{
Eine neue Methode zur Probennahme und Probenaufgabe bei der mit Gaschromatographie/ Massenspektrometrie durchgeführten Luftanalyse im Ultraspurenbereich
}

\author{
Von J. Angerer, A. Haag und G. Lehnert \\ Aus dem Zentralinstitut für Arbeitsmedizin (Direktor: Prof. Dr. med. G. Lehnert) der Freien und Hansestadt \\ Hamburg und dem Institut für organische Chemie der Universität Erlangen-Nürnberg
}

(Eingegangen am 30. August 1974/10. Februar 1975)

\begin{abstract}
Es wird eine neue Methode der Probennahme und Probenaufgabe bei der mit Gaschromatographie/Massenspektrometrie durchgeführten Luftanalyse im Ultraspurenbereich beschrieben. Bei dieser neuen Technik kommt es während der Probenaufgabe zu einer Vortrennung auf der gaschromatographischen Säule, da die bei der Probennahme mitkondensierten Luftbestandteile als Trägergas dienen. Wegen des bei der Probenaufgabe erzeugten Druckgradienten bezeichneten wir die Methode zunächst als „Druckaufgabe“. Dieses Verfahren ist nicht nur von erkenntnistheoretischem Wert. Arbeits- und umweltmedizinische Problemstellungen eröffnen ihm ein breites Anwendungsfeld.
\end{abstract}

\section{A new method for sampling and sample application in the ultra-trace analysis of air by gas chromatography-mass spectrometry}

A new method is described for sampling and sample application in the ultra-trace analysis of air by gas chromatography-mass spectrometry. In this new technique, a preseparation occurs on the gas chromatographic column during sample application, since the atmospheric constituents that are co-condensed during sample application we call this method "Druckaufgabe" (pressure application). The method is not only of theoretical value. It has a potentially wide application to occupational and environmental problems.

Kürzlich haben wir über die Verwendung des Thermogradientrohres (nach Käiser) zur Anreicherung von Spurenkomponenten in Luftproben bei deren gaschromatographisch-massenspektrometrischer Bestimmung berichtet $(1,2)$. Obwohl wir noch keine gezielten Verfeinerungen der Arbeitstechnik im Hinblick auf optimale Anpassung an spezifische Nachweisprobleme ausgearbeitet haben, konnten wir brauchbare qualitative $\mathrm{Er}$ gebnisse erzielen. Dennoch erachten wir es beim augenblicklichen Stand der Technik der Luftanalyse als wünschenswert, auch weitere Verfahren der Probennahme und Probenaufgabe zu überprüfen.

\section{Methodik}

Zum Zwecke der Probenanreicherung haben wir in diesem $\mathrm{Zu}-$ sammenhang das unter anderem von der Gasreinigung her bekannte Verfahren des Ausfrierens von Luftkomponenten untersucht. Ein spiralig gewendeltes Edelstahlrohr (Länge $2 \mathrm{~m}$, Innendurchmesser 3,5 mm, swadgelok-Verschraubung an beiden $\mathrm{En}$ den) wurde zur Probennahme durch Eintauchen in ein Gefäß mit flüssigem Stickstoff auf $-190^{\circ} \mathrm{C}$ gekühlt. Mittels einer Pumpe wurde sofort nach dem Eintauchen ein definiertes Luftvolumen, in unserem Fall 10 Liter, durch diese Kühlfalle gesaugt. Um ein Ansaugen von verdampfendem Stickstoff $\mathrm{zu}$ vermeiden, befand sich auf dem anderen Ende des Rohres ein $2 \mathrm{~m}$ langer Teflonschlauch, der zum Ort der eigentlichen Probenentnahme fuhrte. Eine schematische Darstellung der bei der Probennahme und -aufgabe eingesetzten Gerätekombination zeigt Abbildung 1.

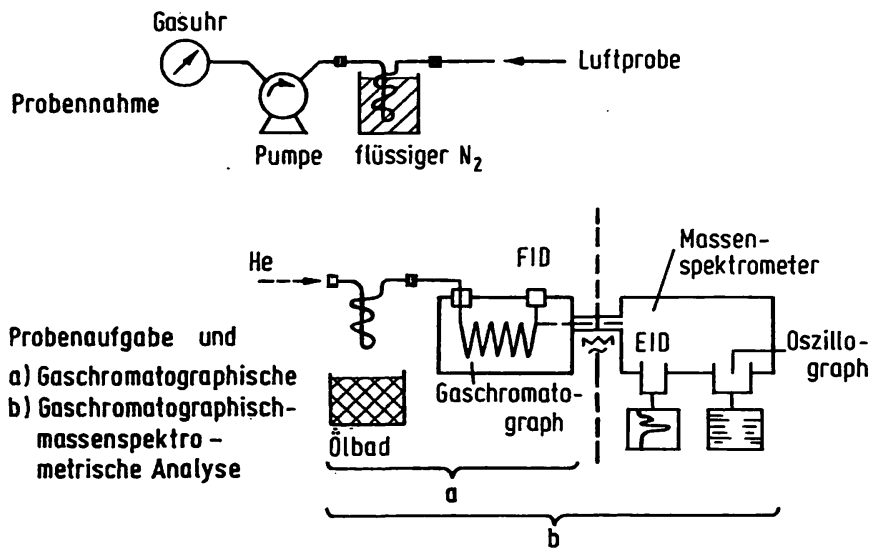

Abb. 1. Schematische Darstellung des apparativen Aufbaues der Probennahme und -aufgabetechnik bei der Luftanalyse mit Gaschromatographie bzw. Gaschromatographie/Massenspektrometrie.

Unter Beibehaltung der Kühlung wurde dieses Ende des Kühlrohres nach dem Entfernen des Teflonschlauches mit einem entsprechend modifizierten Einspritzblock eines Gaschromatographen (statt des verschraubten Scptums wurde ein selbstangefertigtes Übergangsstück verwendet) verbunden (Becker Delft, Typ 409 mit FID). Dann wurde die Pumpe abgekoppelt und das entsprechende Rohrende mit einem Blindflansch dicht verschlossen. Die gaschromatographische Trennsäule wurde vom Flammenionisationsdetektor abgeschraubt, um das GaschromatographieGerät vor dem bei der anschließenden „Druckaufgabe“ entstehenden Druckstoß zu schützen. Nach Entfernung des Kühlbades 
ließen wir das sich auf Raumtemperatur spontan erwärmende Kondensat auf die gekühlte $\left(-150^{\circ} \mathrm{C}\right)$ Trennsäule $(1 / 8$ Zoll Stahlrohr Länge $4 \mathrm{~m} ; 20 \%$ SE 30 auf Chromosorb G-AW/DMCS 80-100 mesh) expandieren. Nach dem Druckausgleich (3 min) wurde die gaschromatographische Säule wieder an den Flammenionisationsdetektor angekoppelt und der Trägergasstrom (Helium $15 \mathrm{ml} / \mathrm{min}$ ) über das Kühlrohr und die Trennsäule geleitet. Durch 5 min Erwärmen der Kondensationsfalle im Trägergasstrom auf $200^{\circ} \mathrm{C}$ wurden auch die höher flüchtigen Komponenten auf die gaschromatographische Trennsäule überführt. Zur Trennung der aufgegebenen Komponenten wurde nun die Trennsäule programmiert von $-150^{\circ} \mathrm{C}$ auf $+250^{\circ} \mathrm{C}$ aufgeheizt. Die Temperaturanstiegsrate betrug $7,5^{\circ} \mathrm{C} / \mathrm{min}$. Ein auf diese Weise gewonnenes FID-Chromatogramm zeigt die Abbildung 2.

Zur qualitativen Identifizierung der getrennten Komponenten wurden in weiteren Versuchen statt des Flammenionisationsdetektors der EID einer Gaschromatographie-Massenspektrometrie-Kombination verwendet. Dazu wurde die gaschromatographische Trennsäule des Becker-Gaschromatographiegerätes über eine beheizbare Kapillare und über den Spaltseperator an den EID des Massenspektroskopiegerätes angeschlossen. Verwendet wurde eine Gaschromatographie-Massenspek trometrieKombination Varian MAT 111 (Firma Varian MAT GmbH Bremen) mit Doppelionenquelle (EID $20 \mathrm{eV}, \mathrm{MSD} 80 \mathrm{eV}$, Betriebstemperatur $200^{\circ} \mathrm{C}$ ). Anhand der mit dem EID erzeugten Leitchromatogramme, die selbstverständlich die gleiche Elu-

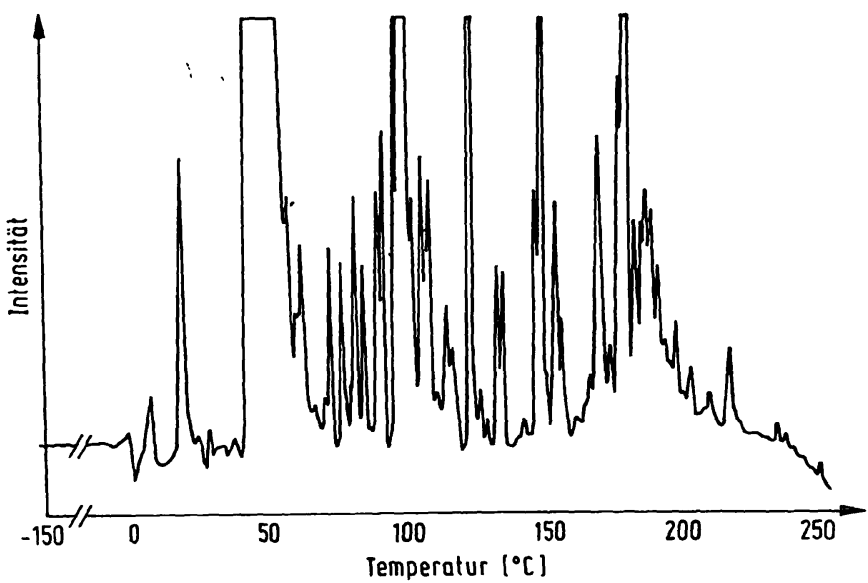

Abb. 2. FID Chromatogramm eines mit der „Druckaufgabe“ aufgebrachten Raumluftkondensats. tionsreihenfolge der Komponenten wie die FID-Chromatogramme aufwiesen, wurden die Massenspektrometrie-Durchläufe der getrennten Komponenten gestartet. Zur Aufzeichnung der Massenspektren diente ein Zweikanal-Galvanometerschreiber (Siemens Oscilloport). Ansonsten wird bei der Verwendung eines Massenspektrometrie-Gerätes anstelle eines FID als Detektor genauso verfahren wie oben beschrieben. In Tabelle 1 sind die massenspektrometrisch anhand ihrer Molekülionen und charakteristischen Fragmentionen identifizierten Substanzen in der Reihenfolge ihrer gaschromatographischen Elutionen angegeben. Die Temperaturangabe bezieht sich auf den Stand des Temperaturprogrammes beim Erscheinen der Kómponente im EID.

\section{Ergebnisse und Diskussion}

Unter dem Begriff „Druckaufgabe“ verstehen wir die stürmisch verlaufende Expansion des Probenkondensats auf die gaschromatographische Säule beim Erwärmen des Probennahmerohres von der Temperatur des flüssigen Stickstoff̣s auf eine höhere Temperatur, in diesem Fall Raumtemperatur, wobei mitkondensierte stark flüchtige Luftbestandteile, wie $\mathrm{O}_{2}, \mathrm{~N}_{2}$ und $\mathrm{CO}_{2}$ als „Treibgase" dienen. Während dịe Hauptmenge des Kondensats mit einem Druckstoß auf die Säule aufgebracht wird, sind Vortrenneffekte aufgrund fraktionierender Destillation aus dem Kondensationsgefäß nur während der Anfangs- und Endphase der Probenaufgabe zu erwarten. Trotzdem dürfte es im Gegensatz zur Thermogradientmethode bereits während der Probenaufgabe zu einer gaschromatographischen Trennung auf der Säule kommen. Die vergleichsweise großen Mengen mitkondensierter Luftbestandteile (Sauerstoff und Stickstoff) wirken nämlich als Trägergas und gestatten bereits bei der Probenaufgabe eine im Vergleich zur Thermogradientrohrtechnik stärkere Auftrennung der einzelnen Komponenten. Diese Auftrennung dürfte weiterhin dadurch verstärkt werden, daß ein Druckgradient entsteht, der zu Beginn der Probenaufgabe große Durchflußraten erzeugt, die bis zum Druckausgleich auf Null absinken.

Tab. 1. Retentionszeiten und Fragmentierung einiger massenspektrometrisch identifizierter Raumluftkomponenten. Standardabweichung (s) und Variabilität (VK) der relativen Retentionen $(\overline{\bar{\alpha}}) . \mathrm{n}=6$.

\begin{tabular}{|c|c|c|c|c|c|c|}
\hline \multirow[t]{3}{*}{ Substanz } & \multicolumn{2}{|l|}{ MSD } & \multicolumn{4}{|l|}{ EID } \\
\hline & \multirow[b]{2}{*}{$\mathrm{M}^{+}[\mathrm{m} / \mathrm{e}]$} & \multirow{2}{*}{$\begin{array}{l}\text { Fragmentionen } \\
{[\mathrm{m} / \mathrm{e}]}\end{array}$} & \multirow{2}{*}{$\begin{array}{l}\text { Elutions- } \\
\text { tempe- } \\
\text { ratur } \\
{\left[{ }^{\circ} \mathrm{C}\right]}\end{array}$} & \multicolumn{2}{|l|}{$\begin{array}{l}\text { relative } \\
\text { Retentionen }\end{array}$} & \multirow{2}{*}{$\begin{array}{l}\text { VK } \\
\text { [\%] }\end{array}$} \\
\hline & & & & $\bar{\alpha}$ & $\mathbf{s}$ & \\
\hline Methanol & 32 & $31,30,29,28$ & 32 & 0,586 & 0,0121 & 2,06 \\
\hline $\mathrm{Cl}_{3} \mathrm{CF}$ & - & $101 / 103 / 105,83,84,86,66 / 68,35 / 37$ & 46 & 0,635 & 0,0086 & 1,35 \\
\hline Aceton & 58 & $43,42,29$ & 51 & 0,640 & 0,0080 & 1,25 \\
\hline Diäthyläther & 74 & $73,59,45,43,29,28$ & 57 & 0,670 & 0,0024 & 0,36 \\
\hline Methylacetat & 74 & $59,43,42,28$ & 63 & 0,681 & 0,0012 & 0,17 \\
\hline Hexan & 86 & $71,57,56,43,42,41,29$ & 84 & 0,748 & 0,0105 & 1,40 \\
\hline Benzol & 78 & $77,52,51,50,39$ & 99 & 0,804 & 0,0010 & 0,13 \\
\hline Trichloräthylen & 130/132/134/136, & $95 / 97 / 99$ & 111 & 0,827 & 0,0101 & 1,22 \\
\hline Toluol & 92 & $91,65,63,51,50$ & 130 & 0,862 & 0,0133 & 1,54 \\
\hline Tetrachloräthylen & $164 / 166 / 168 / 170$ & $129 / 131 / 133,94 / 96 / 98$ & 145 & 0,946 & 0,0014 & 0,15 \\
\hline Xylol & 160 & $105,91,77,76,65,51$ & 162 & 1 & 0,0 & 0,0 \\
\hline
\end{tabular}


In Anbetracht der technisch sicherlich noch nicht perfekten Versuchsanordnung erstaunt neben der erzielten Trennleistung, die unter anderem auf die geschilderten Vorgänge zurückzuführen sein dürfte, hauptsächlich die Reproduzierbarkeit der relativen Retentionszeiten (Tab. 1). Die geschilderten relativ umständlichen Versuchsvorbereitungen, z. B. die zum Schutz der Geräte notwendigen Maßnahmen, lassen sich durch geeignete Modifikation der verwendeten Geräte praktikabler gestalten. (Ein entsprechendes Gerät wird inzwischen von der Firma AMA Analysentechnik, Hilden gebaut). Die mit der „Druckaufgabe“" erhaltenen Ergebnisse sind in dieser Hinsicht den mit der Thermogradient technik erreichten (2) zumindest ebenbürtig, wie aus den geringen Standardabweichungen (s) der auf Xylol bezogenen relativen Retentionen $(\alpha)(3)$ der Einzelkomponenten (Tab. 1) hervorgeht. Darüber hinaus zeigen die mit der „Druckaufgabe" erhaltenen FID-Chromatogramme wesentlich mehr scharfe und gut getrennte Peaks als vergleichbare, mit der Thermogradienttechnik gewonnene Chromatogramme. Dies ist einerseits darauf zurückzuführen, daß im Thermogradientrohr in Abhängigkeit von der Füllung eine selektivere Anreicherung von Spurenkomponenten erzielt wird. Andererseits erlaubt die hier beschriebene Methode eine Anreicherung aus größeren Luftvolumina, da eine Verstopfung durch ausfrierendes Wasser und anderer im Überschuß vorhandener Luftkomponenten im Gegensatz zum Thermogradientrohr u. a. wegen der entfallenden stationären Phase hier nicht zu befürchten ist. Parallel mit der Möglichkeit, größere Probenvolumina anzureichern, dürfte sich die Nachweisgrenze gegenüber den für die Thermogradienttechnik angegebenen (4) um den Faktor 5 bis 10 senken lassen. Eine Diskriminierung einzelner Komponenten sowie eine Beeinträchtigung der Trennschärfe wurden nicht festgestellt.

Von größerer Wichtigkeit scheint uns die Beobachtung, daß bei Verwendung der gleichen gaschromatographischen Trennsäule sowohl bei der Anwendung der Thermogradienttechnik (2) wie bei der „Druckaufgabe“ die Elutionsreihenfolge der Komponenten unverändert bleibt. Das bedeutet, daß die von uns nachgewiesene Vortrennung von Komponenten auf dem Thermogradientrohr (2) offenbar keine entscheidende Rolle für die nachfolgende gaschromatographische Trennung spielt.

Zusammenfassend sehen wir, verglichen mit der Thermogradienttechnik, den Vorteil der „Druckaufgabemethode " in einem größeren qualitativen Informationsgehalt. Dies mag insbesondere für die Analyse arbeitsmedizinisch bedeutsamer niedrig siedender, thermisch instabiler Komponenten gelten. Wegen der verwendeten niedrigen Temperaturen sind hier nämlich im Vergleich zur Thermogradientrohrtechnik keine SekundärReaktionen zu befürchten. Aufbauend auf den mit dieser Methode erhaltenen Ergebnissen läßt sich dann die größere Selektivität der Thermogradientmethode zur gezielten Anreicherung bestimmter interessierender Substanzgruppen sinnvoll ausnützen. Die Methode der Druckaufgabe ist damit nicht nur von erkenntnistheoretischem Wert, obwohl im Hinblick auf die gaschromatographische Trennung durch die als Trägergas dienenden Luftbestandteile und den auftretenden Druckgradienten auch neuartige Trennprinzipien aufgezeigt werden.

Die Technik der Probennahme selbst wird von uns zur Zeit derart modifiziert, daß wir zur Probensammlung nicht mehr - wie hier angegeben - Luft durch eine gekühlte Falle saugen, sondern allein die Wirkung der Volumenkontraktion unter dem Einfluß tiefer Temperaturen zur Probensammlung ausnützen. Diese entscheidende Verbesserung der Probennahmetechnik dürfte das Verfahren noch praxisgerechter gestalten und seine Einsatzmöglichkeiten bei epidemiologischen Studien mit arbeitsmedizinischen und ökotoxikologischen Fragestellungen erleichtern. Gerade bei dem immer dringender werdenden Problem der Summation und Potentierung langfristig einwirkender Schadstoffgemische in subtoxischen Konzentrationen dürfte die beschriebene Technik eine methodische Bereicherung darstellen. Sie unterscheidet sich prinzipiell von anderen in der Literatur geschilderten Verfahren zur Spurenanalyse in der Luft $(1,2,4,5$, 6), wobei es hier zunächst darauf ankam, die qualitative Leistungsfähigkeit der „Druckaufgabe“ zu demonstrieren. Uber unsere Erfahrungen bei der quantitativen Luftanalyse mit dieser Methode wird an anderer Stelle berichtet werden.

\section{Literatur}

1. Angerer, J., Haag, A., Szadkowski, D. \& Lehnert, G. (1973), diese Z. 11, 133-134.

2. Angerer, J. \& Haag, A. (1974), diese Z. 12, 321-326.

3. Jentzsch, D. (1971), Gas Chromatographie, 1. Aufl., S. 17, Frankh'sche Verlagshandlung, Stuttgart.

4. Bergert, K.-H., Betz, V. \& Pruggmayer, D. (1974), Chromatographia 7, 115-121.

5. Kaiser, R. E. (1973), Anal. Chem. 45, 965-967.

6. Kaiser, R. E. (1972), Chromatographia 5, 281-283.
Dipl.-Chem. Dr. Jürgen Angerer Zentralinstitut für Arbeitsmedizin der Freien und Hansestadt Hamburg 2 Hamburg 76,

Adolph-Schönfelder-Straße 5

Postfach 4556 
